\title{
A Case of Curable Dementia Treated by Effective Endovascular Embolization for Dural Arteriovenous Fistula
}

\author{
Tomoyuki Yoshihara $^{a} \quad$ Ryuzaburo Kanazawa $^{a}$ Shinichiro Maeshimab \\ Aiko Osawa $^{c}$ Ikuo Ochiai ${ }^{d}$ Nahoko Uemiya $^{\mathrm{e}}$ Shinya Kohyama $^{\mathrm{e}}$ \\ Fumitaka Yamane $^{e}$ Shoichiro Ishihara ${ }^{e}$ \\ ${ }^{a}$ Department of Neurosurgery, Nagareyama Central Hospital, Nagareyama, ${ }^{b}$ Department \\ of Rehabilitation Medicine II, School of Medicine, Fujita Health University, Toyoake, \\ ${ }^{\mathrm{C}}$ Department of Rehabilitation Medicine, National Center for Geriatrics and Gerontology, \\ Ohbu, ${ }^{\mathrm{d} D e p a r t m e n t}$ of Neurosurgery, Saku Central Hospital, Saku, and ${ }^{\mathrm{e} D e p a r t m e n t}$ of \\ Endovascular Neurosurgery, International Medical Center, Saitama Medical University, \\ Hidaka, Japan
}

\section{Key Words}

Cerebral blood flow - Dementia - Dural arteriovenous fistula - Endovascular treatment . Neuropsychological assessment · Single photon emission computed tomography · Venous hypertension

\begin{abstract}
Background: We report a case in which an effective endovascular treatment cured the worsening of dementia due to a dural arteriovenous fistula (dAVF). Case Report: A 73-yearold woman suffered for 1.5 years from gait disturbance and disorientation that were suspicious of dementia. A brain MRI revealed that her symptoms were likely due to dAVF. An angiography provided a definitive diagnosis of dural transverse-sigmoid sinus fistula. Transvenous embolization completely occluded the fistula and led to a significant improvement of her cognitive function in tandem with a restoration of the cerebral blood flow. Conclusion: Endovascular treatment for dAVF is sometimes effective in treating worsening dementia, which might be due to the drastic change in cerebral blood flow in the area relevant to the cognitive function.

(c) 2014 S. Karger AG, Basel
\end{abstract}

Tomoyuki Yoshihara, MD, PhD

Department of Neurosurgery, Nagareyama Central Hospital

2-132-2, Higashi-Hatsuishi

Nagareyama, Chiba 270-0114 (Japan)

E-Mail t.yoshihara@nch.or.jp 


\section{Introduction}

Dural arteriovenous fistula (dAVF) is a relatively rare cerebral vascular disease, which consists of abnormal arteriovenous shunts within the dura matter. Initial symptoms of dAVF mostly include tinnitus, headache, exophthalmos, and sometimes neurologic deficits with intracranial hemorrhage [1]. However, there are a few reports of dAVF with cognitive impairment [2]. We report a case in which endovascular embolization was effective in treating worsening dementia due to dAVF in tandem with a restoration of cerebral blood flow (CBF).

\section{Case Report}

A 73-year-old right-handed woman suffered from tinnitus, occipital headache, nausea, and anorexia since March 2009. She presented with a slowly worsening dementia, including disorientation to time and place as well as gait disturbance for 1.5 years. By September 2010, she was unable to walk, converse verbally, or perform any activities on her own; her family doctor referred her to a neurologist. T2-weighted MRI showed high intensity in the right middle cerebellar peduncle (fig. 1a), the right globus pallidus (fig. 1b), and the corona radiata. It also revealed remarkable flow void representing dilated cortical veins from the left cerebellum to the bilateral parieto-temporo-occipital lobes. These findings suggested dAVF. She was admitted to our hospital for a thorough examination regarding dAVF, and the patient received endovascular treatment.

On admission, she presented with severe cognitive impairment, including speech disturbance, memory impairment, lack of motivation and apathy. Scores of various neuropsychological tests on initial assessment are shown in table 1. Overall, her neuropsychological assessment showed a severe impairment of her cognitive functions, with a deterioration of her physical activity. Nevertheless, she exhibited no focal signs such as aphasia or hemiparesis.

The study of her cerebral angiography provided a definitive diagnosis for dAVF at the right transverse-sigmoid sinus with severe venous congestion. The right occipital artery, the middle meningeal artery, and the ascending pharyngeal artery were the main feeders (fig. 2a). Although some anterograde blood flow still passed through the severely stenosed sigmoid sinus, most blood flow was in the right transvers sinus run-off retrograde over the confluence, through the straight sinus to the bilateral basal vein of Rosenthal and the inferior sagittal sinus and to the superior petrosal sinus. Especially the reflux of the dilated cortical veins was remarkable in the left cerebellum, medial deep cerebrum, and in the parietotemporo-occipital lobes, suggesting severe venous congestion (Boden type II, Cognard type Ila+b, Larwani Grade 2; fig. 2b, c).

Transvenous embolization with platinum coils delivered through the right transversesigmoid sinus completely occluded the fistula (fig. $2 \mathrm{~d}$ ), which was followed by a significant improvement of her cognitive function with a normalization of CBF. On the first posttreatment day, her cognitive function improved (Mini-Mental State Examination [MMSE] score: 7/30). One month later, her MMSE score had risen to 19/30, and her entire neuropsychological assessment significantly improved, especially in the Wechsler Adult Intelligence Scale III Verbal IQ (81) (table 1). She was able to walk for more than 150 meters without help and could climb and descend stairs. Three weeks after the embolization, threedimensional $99 \mathrm{mTc}$-ethylcysteine dimer single photon emission computed tomography imaging, using an easy Z-score imaging system analysis [3], revealed that the pretreatment 
reduction of $\mathrm{CBF}$ in the bilateral posterior cingulate gyrus, precuneus, and the left parietooccipital cortex (fig. 3a) had improved to normal levels (fig. 3b). On the 38th hospital day, she was transferred to another hospital that emphasized rehabilitation.

Cerebral angiography performed 3 months after embolization confirmed that the dAVF had completely disappeared and that there was no retrograde flow in the cortical veins, and also less venous congestion. Furthermore, 4 months after embolization, her cognitive function progressively improved (as indicated by higher scores on her neuropsychological assessments; table 1).

\section{Discussion}

We succeeded in completely occluding the dAVF through transvenous embolization because of the easy approach to the affected fistula through the ipsilateral sigmoid sinus, where little anterograde flow remained without sinus obliteration. Transvenous embolization with platinum coils appeared to be the most appropriate treatment because of the expected achievement of long-term obliteration of the fistula [4].

The presumed mechanism for the occurrence of worsening dementia from dAVF appears to be venous hypertensive encephalopathy. Hurst et al. [2] reported that angiographic features of dAVF with dementia frequently include reflux of the superior sagittal sinus, straight sinus and cortical veins with transvers/sigmoid sinus occlusion. They noted that high flow through a dAVF triggers a disturbance of the cerebral venous drainage, therefore increasing venous hypertension and brain ischemia, which is implicated as one of the causes of deterioration of cognitive functions such as loss of recent memory, executive functions, and disorientation. Affected patients often present with a worsening of their dementia without any neurological focal signs such as aphasia, paresis, or ataxia [5]. These clinical features applied well to our patient.

In the present case, endovascular treatment might have significantly contributed to a cure for the worsening of dementia by restoring low CBF in the posterior cingulate gyrus, precuneus, and parieto-occipital cortex. It is interesting that a decrease in regional CBF in the parieto-occipital cortex, the posterior cingulate gyrus, and the precuneus is often observed in early-stage Alzheimer's disease [6]. In our patient, a drastic improvement of $\mathrm{CBF}$, relevant to her cognitive functions, might have led to a cure for her dementia. Similar to our case, Waragai et al. [5] reported that endovascular treatment for dAVF could cause CBF to improve in the parieto-occipital cortex, accompanied by a recovery from the cognitive dysfunction. Few reports have considered the possible association of the regional CBF pattern with dAVF-dementia.

It is often difficult to treat cognitive impairment deriving from neurodegenerative disorders such as Alzheimer's disease, Parkinson's disease, or most types of cerebrovascular dementia. However, cognitive dysfunctions due to dAVF might be curable. Like in our patient's case, it is important to recognize treatable dementia. We might often miss that in our daily clinical practice.

\section{Disclosure Statement}

The authors declare that they have no conflicts of interest. 


\section{References}

1 Obrador S, Soto M, Silvela J: Clinical syndromes of arteriovenous malformations of the transverse-sigmoid sinus. J Neurol Neurosurg Psychiatry 1975;38:436-451.

-2 Hurst RW, Bagley LJ, Galetta S, Glosser G, Lieberman AP, Trojanowski J, Sinson G, Stecker M, Zager E, Raps EC, Flamm ES: Dementia resulting from dural arteriovenous fistulas: the pathologic findings of venous hypertensive encephalopathy. AJNR Am J Neuroradiol 1998;19:1267-1273.

-3 Kanetaka H, Matsuda H, Asada T, Ohnishi T, Yamashita F, Imabayashi E, Tanaka F, Nakano S, Takasaki M: Effects of partial volume correction on discrimination between very early Alzheimer's dementia and controls using brain perfusion SPECT. Eur J Nucl Med Mol Imaging 2004;31:975-980.

4 Wong GK, Poon WS, Yu SC, Zhu CX: Transvenous embolization for dural transverse sinus fistulas with occluded sigmoid sinus. Acta Neurochir (Wien) 2007;149:929-935.

-5 Waragai M, Takeuchi H, Fukushima T, Haisa T, Yonemitsu T: MRI and SPECT studies of dural arteriovenous fistulas presenting as pure progressive dementia with leukoencephalopathy: a cause of treatable dementia. Eur J Neurol 2006;13:754-759.

-6 Waragai M, Yamada T, Matsuda H: Evaluation of brain perfusion SPECT using an easy Z-score imaging system (eZIS) as an adjunct to early-diagnosis of neurodegenerative diseases. J Neurol Sci 2007;260:57-64.

Table 1. Results of neuropsychological tests before and after endovascular treatment

\begin{tabular}{|c|c|c|c|}
\hline & Before surgery & $\begin{array}{l}1 \text { month after } \\
\text { surgery }\end{array}$ & $\begin{array}{l}4 \text { months after } \\
\text { surgery }\end{array}$ \\
\hline MMSE (30) & $1 / 30$ & $19 / 30$ & $24 / 30$ \\
\hline Digit span (forward/backward) & $3 / 0$ & $6 / 2$ & $5 / 4$ \\
\hline \multicolumn{4}{|l|}{ Auditory Verbal Learning Test (15) } \\
\hline Immediate & $0,0,0,0,0 / 15$ & $4,5,6,9,11 / 15$ & $5,8,8,9,10 / 15$ \\
\hline Recognition & $0 / 15$ & $10 / 15$ & $14 / 15$ \\
\hline Delayed & $0 / 15$ & $7 / 15$ & $9 / 15$ \\
\hline Raven's progressive matrices (36) & $0 / 36$ & $23 / 36$ & $29 / 36$ \\
\hline Frontal Assessment Battery (18) & $3 / 18$ & $11 / 18$ & $13 / 18$ \\
\hline \multicolumn{4}{|l|}{ Wechsler Adult Intelligence Scale III } \\
\hline Verbal IQ & 48 & 81 & 84 \\
\hline Performance IQ & 49 & 72 & 87 \\
\hline Full IQ & 42 & 74 & 84 \\
\hline Verbal comprehension & $<50$ & 92 & 92 \\
\hline Visual perception & $<50$ & 81 & 83 \\
\hline Working memory & $<50$ & 74 & 88 \\
\hline Process speed & 50 & 81 & 100 \\
\hline \multicolumn{4}{|l|}{ Wechsler Memory Scale-Revised } \\
\hline Verbal memory & 50 & 80 & 86 \\
\hline Visual memory & $<50$ & 68 & 102 \\
\hline General memory & $<50$ & 74 & 90 \\
\hline Concentration/attention & $<50$ & 81 & 81 \\
\hline Delayed recall & $<50$ & 73 & 88 \\
\hline \multicolumn{4}{|l|}{ Functional Independence Measure } \\
\hline Motor (91) & $27 / 91$ & $66 / 91$ & $88 / 91$ \\
\hline Cognitive (35) & $7 / 35$ & $25 / 35$ & $31 / 35$ \\
\hline Total & 34 & 91 & 119 \\
\hline
\end{tabular}




\section{Case Reports in Neurology}
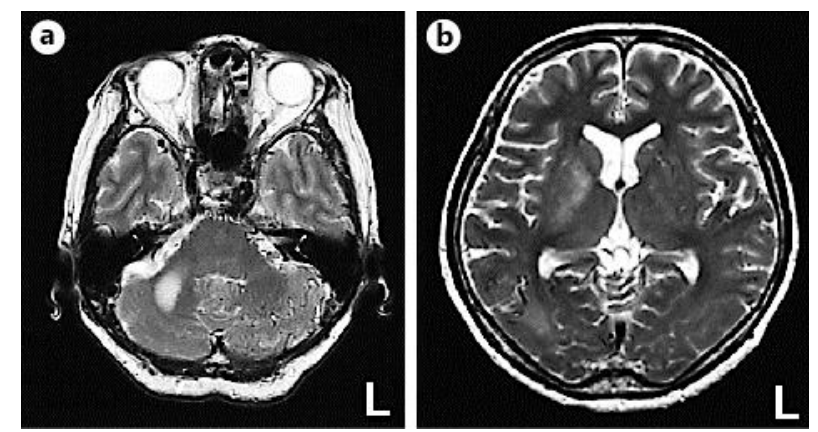

Fig. 1. Brain MRI at initial diagnosis. MRI images show high intensity in the right middle cerebellar peduncle (a) and the globus pallidus (b). Dilated cortical veins are revealed as a flow void from the left cerebellum to the bilateral parieto-temporo-occipital lobe $(\mathbf{a}, \mathbf{b})$.
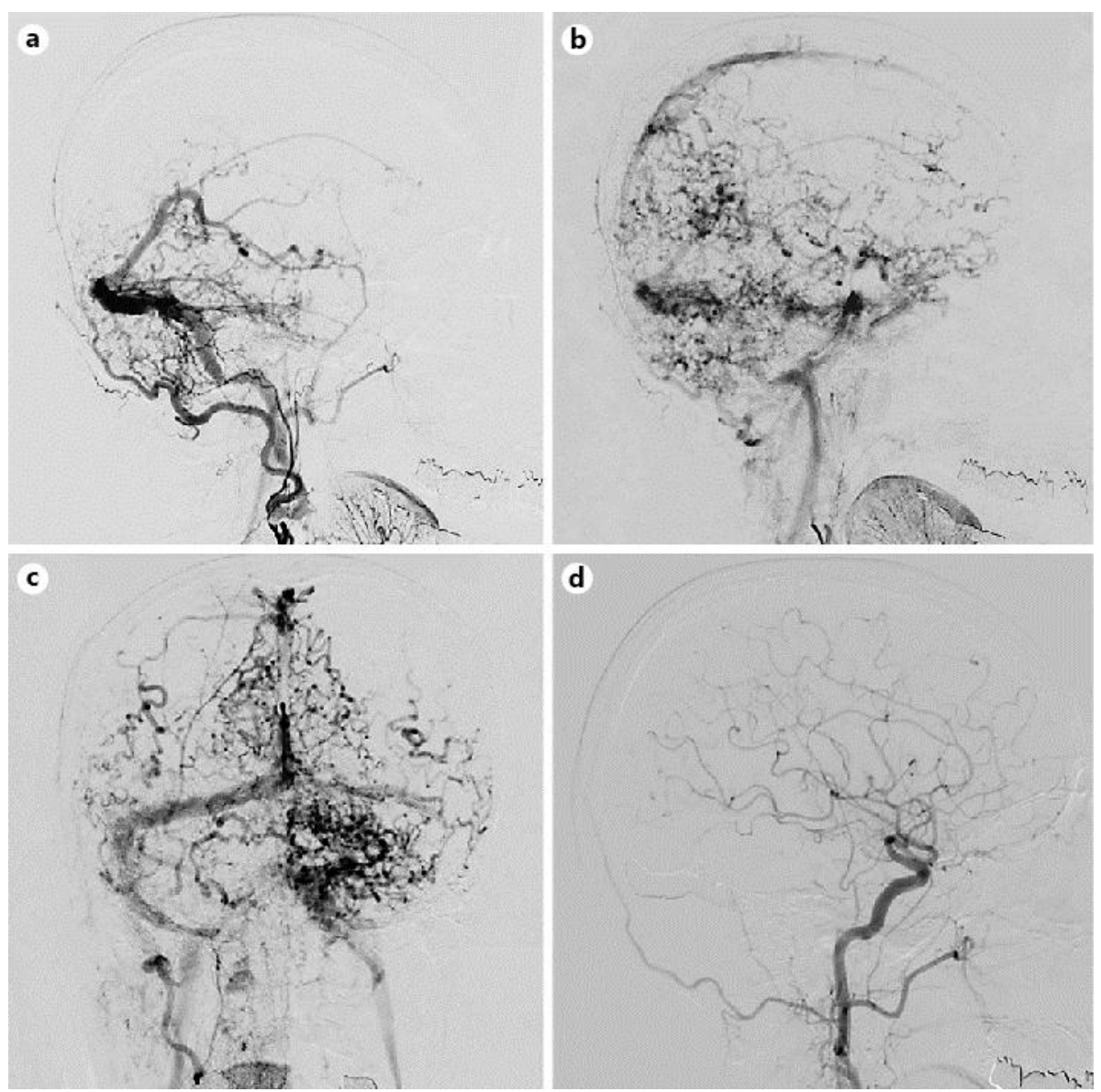

Fig. 2. Right external carotid arteriogram in the lateral (a, b) and anterior-posterior views (c) confirming the definitive diagnosis of dural transverse-sigmoid sinus fistula; the right common carotid arteriogram shows a complete obliteration of the fistula after endovascular treatment (d). Early arterial phase of the external carotid arteriogram shows the right occipital artery, middle meningeal artery, and the ascending pharyngeal artery as main feeders in (a). In the late arterial phase, most drainage flow runs off retrograde from the straight sinus to the basal vein of Rosenthal and to the inferior sagittal sinus, with a severe venous congestion in the cerebellum, medial deep cerebrum, and parieto-temporo-occipital lobe (b, c). 
Yoshihara et al:: A Case of Curable Dementia Treated by Effective Endovascular Embolization for Dural Arteriovenous Fistula
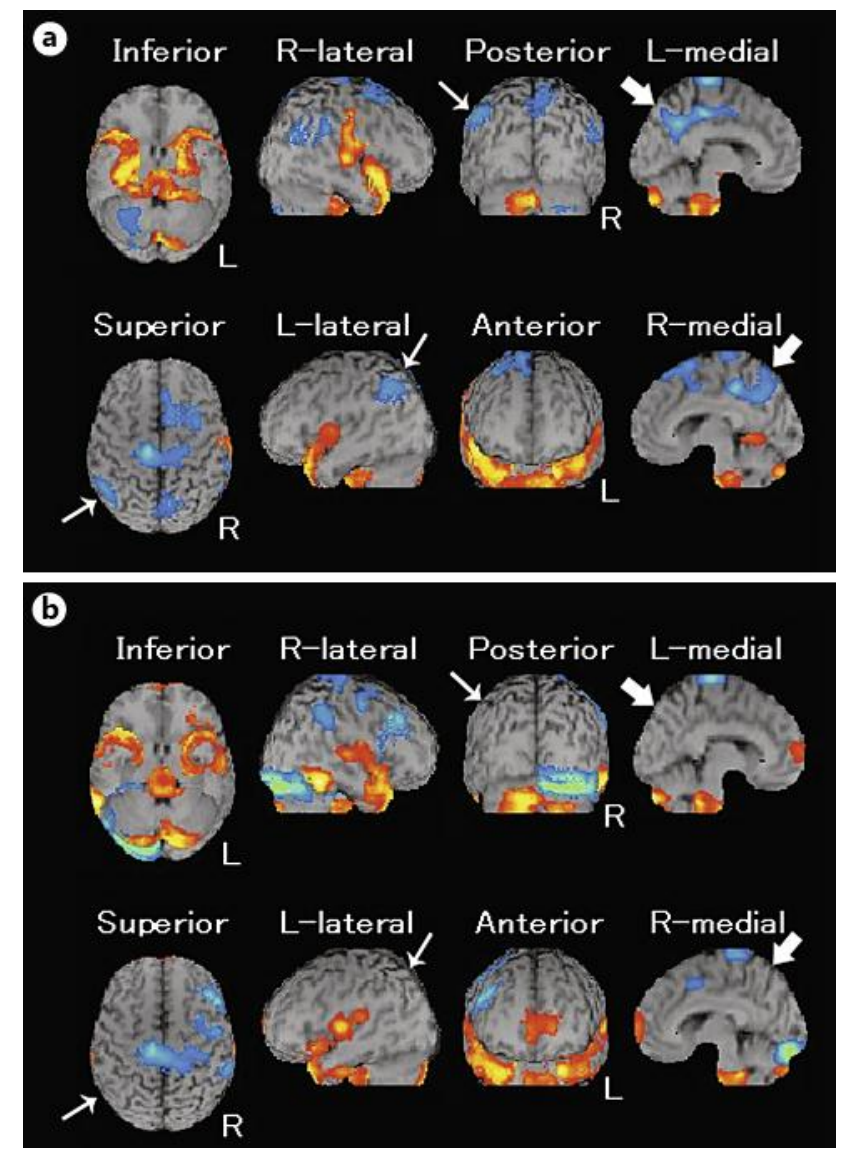

Fig. 3. Three-dimensional 99mTc-ethylcysteine dimer SPECT imaging before (a) and after (b) endovascular embolization. The relative reduction of cerebral blood flow in the bilateral posterior cingulate gyrus, precuneus (broad arrows) and the left parieto-occipital cortex (narrow arrows) (a) has improved to a normal extent after treatment (b). 